\title{
Integrating job parallelism in real-time scheduling theory*
}

\author{
Sébastien Collette $^{\dagger} \quad$ Liliana Cucu ${ }^{\dagger} \quad$ Joël Goossens $^{\dagger} \mathbb{I}$
}

\begin{abstract}
We investigate the global scheduling of sporadic, implicit deadline, real-time task systems on multiprocessor platforms. We provide a task model which integrates job parallelism. We prove that the time-complexity of the feasibility problem of these systems is linear relatively to the number of (sporadic) tasks for a fixed number of processors. We propose a scheduling algorithm theoretically optimal (i.e., preemptions and migrations neglected). Moreover, we provide an exact feasibility utilization bound. Lastly, we propose a technique to limit the number of migrations and preemptions.
\end{abstract}

\section{Introduction}

The use of computers to control safety-critical real-time functions has increased rapidly over the past few years. As a consequence, real-time systems - computer systems where the correctness of each computation depends on both the logical results of the computation and the time at which these results are produced - have become the focus of much study. Since the concept of "time" is of such importance in real-time application systems, and since these systems typically involve the sharing of one or more resources among various contending processes, the concept of scheduling is integral to real-time system design and analysis. Scheduling theory as it pertains to a finite set of requests for resources is a well-researched topic. However, requests in real-time environments are often of a recurring nature. Such systems are typically modeled as finite collections of simple, highly repetitive tasks, each of which generates jobs in a very predictable manner. These tasks have bounds upon their worst-case execution requirements and their periods, and associated deadlines.

In this work, we consider sporadic task systems, i.e., where there is at least $T_{i}$ (called the period) time units between two consecutive job instances of the same task. A job which occurs at time $t$ must be executed for at most $C_{i}$ time units in the time interval $\left[t, t+D_{i}\right.$ ) (where $D_{i}$ is the relative deadline). A particular case of sporadic tasks are the periodic ones for which the period is the exact temporal separation between the arrival of two successive jobs generated by the task. We

*This is an extended version of the paper Integrating Job Parallelism in Real-Time Scheduling Theory, published in the journal Information Processing Letters. Ben Rodriguez ${ }^{\dagger}$ pointed that the definition of work-limited parallelism did not express constraints that we were using implicitly in the remainder of the paper. Consequently we adapted the definition and clarified the proof.

${ }^{\dagger}$ Computer Science Department, Université Libre de Bruxelles, CP212, Bvd. du Triomphe, 1050 Brussels, Belgium

‡Chargé de recherches du F.R.S.-FNRS, sebastien.collette@ulb.ac.be

\$liliana.cucu@ulb.ac.be

q joel.goossens@ulb.ac.be. 
shall distinguish between implicit deadline systems where $D_{i}=T_{i}, \forall i$; constrained deadline systems where $D_{i} \leq T_{i}, \forall i$; and arbitrary deadline systems where there is no constraint between the deadline and the period. Moreover, we assume that the various tasks are independent (i.e., except the $m$ processors there are no other shared resources, no critical sections nor precedence constraints).

The scheduling algorithm determines which job[s] should be executed at each time instant. We distinguish between off-line and on-line schedulers. On-line schedulers construct the schedule during the execution of the system; while off-line schedulers mimic during the execution of the system a precomputed (off-line) schedule. Remark that if a task is not active at a given time instant and the off-line schedule planned to execute that task on a processor, the latter is simply idled (or used for a non-critical task).

When there is at least one schedule satisfying all constraints of the system, the system is said to be feasible. More formal definitions of these notions are given in Section 2.

Uniprocessor sporadic (and periodic) real-time systems are well studied since the seminal paper of Liu and Layland [12] which introduces a model of implicit deadline systems. For uniprocessor systems we know that the worst case arrival pattern for sporadic tasks corresponds to the one of (synchronous and) periodic tasks (see, e.g. [14]). Consequently, most of the results obtained for periodic tasks remain for sporadic ones. Unfortunately, this is not the case upon multiprocessors due to scheduling anomalies (see, e.g. [1]).

The literature considering scheduling algorithms and feasibility tests for uniprocessor scheduling is tremendous. In contrast for multiprocessor parallel machines the problem of meeting timing constraints is a relatively new research area.

\subsection{Model}

We deal with jobs which may be executed on different processors at the very same instant, in which case we say that job parallelism is allowed. For a task $\tau_{i}$ and $m$ identical processors we define a $m$ tuple of real numbers $\Gamma_{i} \stackrel{\text { def }}{=}\left(\gamma_{i, 1}, \ldots, \gamma_{i, m}\right)$ with the interpretation that a job of $\tau_{i}$ that executes for $t$ time units on $j$ processors completes $\gamma_{i, j} \times t$ units of execution. Full parallelism, which corresponds to the case where $\Gamma_{i}=(1,2, \ldots, m)$ is not realistic; moreover, if full parallelism is allowed the multiprocessor scheduling problem is equivalent to the uniprocessor one (by considering, e.g., a unique processor $m$ times faster).

In this work, we consider work-limited job parallelism with the following definition:

Definition 1 (work-limited parallelism). The job parallelism is said to be work-limited if and only if for all $\Gamma_{i}$ we have:

$$
\begin{gathered}
\forall 1 \leq i \leq n, \forall 1 \leq j<j^{\prime} \leq m, \\
\frac{j^{\prime}}{j}>\frac{\gamma_{i, j^{\prime}}}{\gamma_{i, j}} \text { and } \\
\gamma_{i,\left(j^{\prime}+1\right)}-\gamma_{i, j^{\prime}} \leq \gamma_{i,(j+1)}-\gamma_{i, j}
\end{gathered}
$$

Note that the last restriction is equivalent to

$$
\frac{\gamma_{i,\left(j^{\prime}+c\right)}-\gamma_{i, j^{\prime}}}{c} \leq \frac{\gamma_{i,(j+d)}-\gamma_{i, j}}{d}
$$

For instance, the $m$-tuple $\Gamma_{i}=(1.0,1.1,1.2,1.3,4.9)$ is not a work-limited job parallelism, since $\gamma_{i, 5}=4.9>1.3 \times \frac{5}{4}=1.625$. These restrictions may at first seem strong, but are in fact intuitive: 
we require that parallelism cannot be achieved for free, and that even if adding one processor decreases the time to finish a parallel job, a parallel job on $j^{\prime}$ processors will never run $j^{\prime} / j$ times as fast as on $j$ processors. Moreover, if going from $j$ to $j+1$ processors implies some performance loss, then going from $j+1$ to $j+2$ processors must impact the performance by at least the same amount 1 .

Many applications fit in this model, as the increase of parallelism often requires more time to synchronize and to exchange data between parallel processes. Remark that work-limited parallelism requires that for each task (say $\left.\tau_{i}\right)$, the quantities $\gamma_{i, j}$ are distinct $\left(\gamma_{i, 1}<\gamma_{i, 2}<\gamma_{i, 3}<\cdots\right)$.

\subsection{Related research}

Even if the multiprocessor scheduling of sporadic task systems is a new research field, important results have already been obtained. See, e.g., [2, 4, 3, 15, 7] for details.

All these works consider models of tasks where jobs use at most a single processor each time instant. This restriction is natural for the uniprocessor scheduling since only one processor is available at any time instant even if we deal with parallel algorithms. Nowadays, the use of parallel computing is growing (see, e.g., [11]); moreover, parallel programs can be easily designed using the Message Passing Interface (MPI [8, 9]) or the Parallel Virtual Machine (PVM [16, 6]) paradigms. Even better, sequential programs can be parallelized using tools like OpenMP (see [5] for details). Therefore for the multiprocessor case we should be able to describe jobs that may be executed on different processors at the same time instant. For instance, we find such requirements in real-time applications such as robot arm dynamics [17], where the computation of dynamics and the solution of a linear systems are both parallelizable and contain real-time constraints.

Few models and results in the literature concern real-time systems taking into account job parallelism. Manimaran et al. in [13] consider the non-preemptive EDF scheduling of periodic tasks, moreover they consider moldable tasks (the actual number of used processors is determined before starting the system and remains unchanged), while we consider malleable tasks (the number of assigned processors to a task may change during the execution). Meanwhile, their task model and parallelism restriction (i.e., the sub-linear speedup) is quite similar to our model and our parallelism restriction (work-limited). Han et al. in [10] considered the scheduling of a (finite) set of real-time jobs allowing job parallelism. Their scheduling problem is quite different than our, moreover they do not provide a real model to take into account the parallelism.

\subsection{This research}

In this paper, we deal with global scheduling ${ }^{2}$ of implicit deadline sporadic task systems with worklimited job parallelism upon identical parallel machines, i.e., where all the processors are identical in the sense that they have the same computing power. We consider the feasibility problem of these systems, taking into account work-limited job parallelism. For work-limited job parallelism we prove that the time-complexity of the feasibility problem is linear relative to the number of tasks for a fixed number of processors. We provide a scheduling algorithm.

To the best of our knowledge there is no such result in the literature and this manuscript provides a model, a first feasibility test and a first exact utilization bound for such kind of systems.

\footnotetext{
${ }^{1}$ Ben Rodriguez pointed out that we implicitly use this last restriction in the remainder of the paper, and that it is thus required in the definition of work-limited parallelism.

${ }^{2}$ Job migration and preemption are allowed.
} 


\subsection{Organization}

This paper is organized as follows. In Section 2, we introduce our model of computation. In Section 3, we present the main result for the feasibility problem of implicit deadline sporadic task systems with work-limited job parallelism upon identical parallel machines when global scheduling is used. We prove that the time-complexity of the feasibility problem is linear relative to the number of tasks when the number of processors is fixed. We provide a linear scheduling algorithm which is proved theoretically optimal and we give an exact feasibility utilization bound. In Section 4, we propose a technique to limit the number of migrations and preemptions. We conclude and we give some hints for future work in Section 5 .

\section{Definitions and assumptions}

We consider the scheduling of sporadic task systems on $m$ identical processors $\left\{p_{1}, p_{2}, \ldots, p_{m}\right\}$. A task system $\tau$ is composed of $n$ sporadic tasks $\tau_{1}, \tau_{2}, \ldots, \tau_{n}$, each task is characterized by a period (and implicit deadline) $T_{i}$, a worst-case execution time $C_{i}$ and a $m$-tuple $\Gamma_{i}=\left(\gamma_{i, 1}, \gamma_{i, 2}, \ldots, \gamma_{i, m}\right)$ to describe the job parallelism.

We assume that $\gamma_{i, 0} \stackrel{\text { def }}{=} 0(\forall i)$ in the following. A job of a task can be scheduled at the very same instant on different processors. In order to define the degree of parallelization of each task $\tau_{i}$ we define the execution ratios $\gamma_{i, j}, \forall j \in\{1,2, \ldots, m\}$ associated to each task-index of processor pair. A job that executes for $t$ time units on $j$ processors completes $\gamma_{i, j} \times t$ units of execution. In this paper we consider work-limited job parallelism as given by Definition 1 .

We will use the notation $\tau_{i} \stackrel{\text { def }}{=}\left(C_{i}, T_{i}, \Gamma_{i}\right), \forall i$ with $\Gamma_{i}=\left(\gamma_{i, 1}, \gamma_{i, 2}, \ldots, \gamma_{i, m}\right)$ with $\gamma_{i, 1}<\gamma_{i, 2}<\cdots<$ $\gamma_{i, m}$. Such a sporadic task generates an infinite sequence of jobs with $T_{i}$ being a lower bound on the separation between two consecutive arrivals, having a worst-case execution requirement of $C_{i}$ units, and an implicit relative hard deadline $T_{i}$. We denote the utilization of $\tau_{i}$ by $u_{i} \stackrel{\text { def }}{=} \frac{C_{i}}{T_{i}}$. In our model, the period and the worst-case execution time are integers.

A task system $\tau$ is said to be feasible upon a multiprocessor platform if under all possible scenarios of arrivals there exists at least one schedule in which all tasks meet their deadlines.

\subsection{Minimal required number of processors}

Notice that a task $\tau_{i}$ requires more than $k$ processors simultaneously if $u_{i}>\gamma_{i, k}$; we denote by $k_{i}$ the largest such $k$ (meaning that $k_{i}$ is the smallest number of processor[s] such that the task $\tau_{i}$ is schedulable on $k_{i}+1$ processors):

$$
k_{i} \stackrel{\text { def }}{=} \begin{cases}0 & \text { if } u_{i} \leq \gamma_{i, 1} \\ \max _{k=1}^{m}\left\{k \mid \gamma_{i, k}<u_{i}\right\} & \text { otherwise. }\end{cases}
$$

For example, let us consider the task system $\tau=\left\{\tau_{1}, \tau_{2}\right\}$ to be scheduled on three processors. We have $\tau_{1}=\left(6,4, \Gamma_{1}\right)$ with $\Gamma_{1}=(1.0,1.5,2.0)$ and $\tau_{2}=\left(3,4, \Gamma_{2}\right)$ with $\Gamma_{2}=(1.0,1.2,1.3)$. Notice that the system is infeasible if job parallelism is not allowed since $\tau_{1}$ will never meet its deadline unless it is scheduled on at least two processors (i.e., $k_{1}=1$ ). There is a feasible schedule if the task $\tau_{1}$ is scheduled on two processors and $\tau_{2}$ on a third one (i.e., $k_{2}=0$ ). 


\subsection{Canonical schedule}

Definition 2 (schedule $\sigma$ ). For any task system $\tau=\left\{\tau_{1}, \ldots, \tau_{n}\right\}$ and any set of $m$ processors $\left\{p_{1}, \ldots, p_{m}\right\}$ we define the schedule $\sigma(t)$ of system $\tau$ at instant $t$ as $\sigma: \mathbb{R}_{+} \rightarrow\{0,1, \ldots, n\}^{m}$ where $\sigma(t) \stackrel{\text { def }}{=}\left(\sigma_{1}(t), \sigma_{2}(t), \ldots, \sigma_{m}(t)\right)$ with

$$
\sigma_{j}(t) \stackrel{\text { def }}{=} \begin{cases}0 & \text { if there is no task scheduled on } p_{j} \text { at instant } t \\ i & \text { if } \tau_{i} \text { is scheduled on } p_{j} \text { at instant } t\end{cases}
$$

for all $1 \leq j \leq m$.

We will now define canonical schedules. In what follows we will prove that it is always possible to find such a canonical schedule for all feasible task systems (see Theorem 5). Refer to Figure 2 for an example of a simple canonical schedule. Intuitively a schedule is canonical if it is a schedule where the tasks with higher indices are assigned to the highest available processors greadily.

Definition 3 (canonical schedule). For any task system $\tau=\left\{\tau_{1}, \ldots, \tau_{n}\right\}$ and any set of $m$ processors $\left\{p_{1}, \ldots, p_{m}\right\}$, a schedule $\sigma$ is canonical if and only if the following inequalities are satisfied:

$$
\begin{gathered}
\forall 1 \leq j \leq m, \forall 0 \leq t<t^{\prime}<1: \sigma_{j}\left(t^{\prime}\right) \leq \sigma_{j}(t) \\
\forall 1 \leq j<j^{\prime} \leq m, \forall t, t^{\prime} \in[0,1): \sigma_{j}(t) \leq \sigma_{j^{\prime}}\left(t^{\prime}\right)
\end{gathered}
$$

and the schedule $\sigma$ contains a pattern that is repeated every unit of time, i.e., $\forall t \in \mathbb{R}_{+}, \forall 1 \leq j \leq m$ : $\sigma_{j}(t)=\sigma_{j}(t+1)$.

Without loss of generality for the feasibility problem, we consider a feasibility interval of length 1. Notice that the following results can be generalized to consider any interval of length $\ell$, as long as $\ell$ divides entirely the period of every task.

\section{Our feasibility problem}

In this section we prove that if a task system $\tau$ is feasible, then there exists a canonical schedule in which all tasks meet their deadlines. We give an algorithm which, given any task system, constructs the canonical schedule or answers that no schedule exists. The algorithm runs in $O(n)$ time with $n$ the number of tasks in the system.

We start with a generic necessary condition for schedulability using work-limited parallelism:

Theorem 4. In the work-limited parallelism model and using an off-line scheduling algorithm, a necessary condition for a sporadic task system $\tau$ to be feasible on $m$ processors is given by:

$$
\sum_{i=1}^{n}\left(k_{i}+\frac{u_{i}-\gamma_{i, k_{i}}}{\gamma_{i, k_{i}+1}-\gamma_{i, k_{i}}}\right) \leq m
$$


Proof. As $\tau$ is feasible on $m$ processors, there exists a schedule $\sigma$ meeting every deadline. We consider any time interval $[t, t+P)$ with $P \stackrel{\text { def }}{=} \operatorname{lcm}\left\{T_{1}, T_{2}, \ldots, T_{n}\right\}$.

Let $a_{i, j}$ denote the duration where jobs of a task $\tau_{i}$ are assigned to $j$ processors on the interval $[t, t+P)$ using the schedule $\sigma$. The sum $\sum_{j=1}^{m} j \cdot a_{i, j}$ gives the total processor use of the task $\tau_{i}$ on the interval (total number of time units for which a processor has been assigned to $\tau_{i}$ ). As we can use at most $m$ processors concurrently, we know that

$$
\sum_{i=1}^{n} \sum_{j=1}^{m} j \cdot a_{i, j} \leq m \cdot P
$$

otherwise the jobs are assigned to more than $m$ processors on the interval. If on some interval of length $\ell, \tau_{i}$ is assigned to $j$ processors, we can achieve the same quantity of work on $j^{\prime}>j$ processors on an interval of length $\ell \frac{\gamma_{i, j}}{\gamma_{i, j^{\prime}}}$. In the first case, the processor use of the task $i$ is $\ell j$, while in the second case it is $\ell j^{\prime} \frac{\gamma_{i, j}}{\gamma_{i, j^{\prime}}}$. By the first restriction that we enforced on the tuple $\Gamma_{i}$ (see Definition 1), we have

$$
\begin{aligned}
\ell j^{\prime} \frac{\gamma_{i, j}}{\gamma_{i, j^{\prime}}} & >\ell j^{\prime} \frac{\gamma_{i, j^{\prime} j^{\prime}}}{\gamma_{i, j^{\prime}}} \\
& >\ell j
\end{aligned}
$$

Let $\sigma^{\prime}$ be a slightly modified schedule compared to $\sigma$, where $\forall i \neq i^{\prime}, \forall j, a_{i, j}^{\prime}=a_{i, j}$. For the task $\tau_{i^{\prime}}$, it is scheduled on $j^{\prime}$ processors instead of $j<j^{\prime}$ in $\sigma$ for some interval of length $\ell$, i.e.

$$
\begin{gathered}
a_{i^{\prime}, j}^{\prime}=a_{i^{\prime}, j}-\ell \\
a_{i^{\prime}, j^{\prime}}^{\prime}=a_{i^{\prime}, j^{\prime}}+\ell \frac{\gamma_{i, j}}{\gamma_{i, j^{\prime}}}
\end{gathered}
$$

Then, for that task $\tau_{i^{\prime}}$,

$$
\sum_{j=1}^{m} j \cdot a_{i^{\prime}, j}^{\prime}>\sum_{j=1}^{m} j \cdot a_{i^{\prime}, j}
$$

This proves that increasing the parallelism yields an increased sum. It remains to prove that it is not better to increase the parallelism to $j^{\prime}>j$ processors on some interval $\ell$ in order to decrease it to $j^{\prime \prime}<j$ processors on some other interval $\ell^{\prime}$ (see Figure 1).

The quantity of work originally achieved on the interval $\ell+\ell^{\prime}$ is $\left(\ell+\ell^{\prime}\right) \cdot \gamma_{i, j}$ while the processor use is $\left(\ell+\ell^{\prime}\right) \cdot j$. After the change, the quantity of work is $\ell \gamma_{i, j^{\prime}}+\ell^{\prime} \gamma_{i, j^{\prime \prime}}$ for a processor use of $\ell j^{\prime}+\ell^{\prime} j^{\prime \prime}$.

Suppose the processor use is not changed, and we shall show that the quantity of work has decreased. We start by noting that as the processor use is not changed, $\left(\ell+\ell^{\prime}\right) \cdot j=\ell j^{\prime}+\ell^{\prime} j^{\prime \prime}$, and we get

$$
\ell\left(j^{\prime}-j\right)=\ell^{\prime}\left(j-j^{\prime \prime}\right)
$$



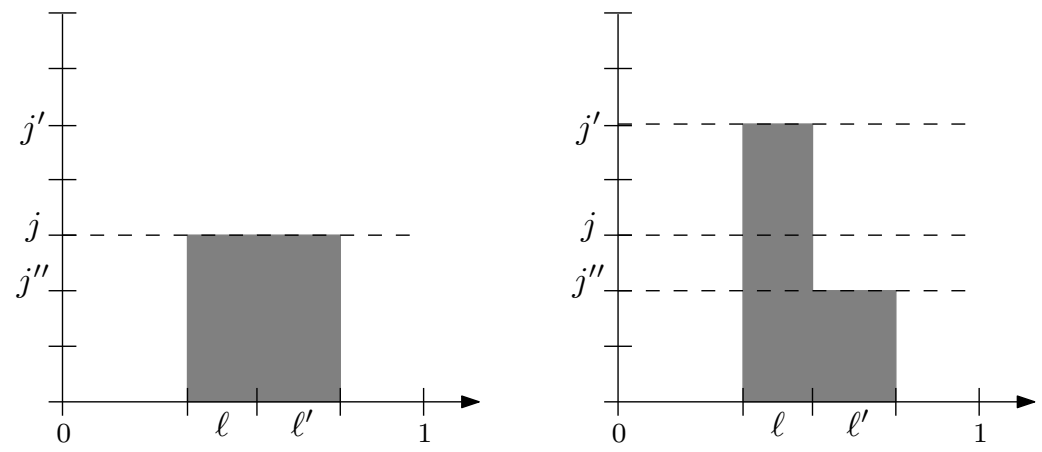

Figure 1: Increasing the parallelism on some interval $\ell$ to be able to decrease it on $\ell^{\prime}$.

From the last restriction enforced by work-limited parallelism (see Definition 1), we know that

$$
\gamma_{i, j^{\prime}}-\gamma_{i, j} \leq\left(\gamma_{i, j}-\gamma_{i, j^{\prime \prime}}\right) \cdot \frac{j^{\prime}-j}{j-j^{\prime \prime}}
$$

Now we have all the tools needed:

$$
\begin{aligned}
\ell \gamma_{i, j^{\prime}}+\ell^{\prime} \gamma_{i, j^{\prime \prime}} & =\ell\left(\gamma_{i, j^{\prime}}-\gamma_{i, j}\right)+\ell \gamma_{i, j}+\ell^{\prime} \gamma_{i, j}-\ell^{\prime}\left(\gamma_{i, j}-\gamma_{i, j^{\prime \prime}}\right) \\
& =\ell\left(\gamma_{i, j^{\prime}}-\gamma_{i, j}\right)-\ell^{\prime}\left(\gamma_{i, j}-\gamma_{i, j^{\prime \prime}}\right) \frac{j-j^{\prime \prime}}{j-j^{\prime \prime}}+\left(\ell+\ell^{\prime}\right) \gamma_{i, j} \\
& =\ell\left(\gamma_{i, j^{\prime}}-\gamma_{i, j}\right)-\ell\left(\gamma_{i, j}-\gamma_{i, j^{\prime \prime}}\right) \frac{j^{\prime}-j}{j-j^{\prime \prime}}+\left(\ell+\ell^{\prime}\right) \gamma_{i, j} \text { (by Equation 2) } \\
& \leq \ell\left(\gamma_{i, j}-\gamma_{i, j^{\prime \prime}}\right) \frac{j^{\prime}-j}{j-j^{\prime \prime}}-\ell\left(\gamma_{i, j}-\gamma_{i, j^{\prime \prime}}\right) \frac{j^{\prime}-j}{j-j^{\prime \prime}}+\left(\ell+\ell^{\prime}\right) \gamma_{i, j} \text { (by Equation 3) } \\
& \leq\left(\ell+\ell^{\prime}\right) \gamma_{i, j}
\end{aligned}
$$

In other words we decreased the quantity of work for a fixed amount of processor use; if we want to keep the same quantity of work, we need to increase the processor use and thus the sum defined above.

So, we proved that we should minimize the parallelism; as we want to derive a necessary condition, we schedule the task on the minimal number of processors required. A lower bound on the sum is then given by

$$
k_{i} \cdot P+\frac{u_{i}-\gamma_{i, k_{i}}}{\gamma_{i, k_{i}+1}-\gamma_{i, k_{i}}} \cdot P
$$

which corresponds to scheduling the task on $k_{i}+1$ processors for a minimal amount of time, and on $k_{i}$ processors for the rest of the interval. Then

$$
\sum_{j=1}^{m} j \cdot a_{i, j} \geq k_{i} \cdot P+\frac{u_{i}-\gamma_{i, k_{i}}}{\gamma_{i, k_{i}+1}-\gamma_{i, k_{i}}} \cdot P
$$


and thus

$$
\begin{aligned}
\sum_{i=1}^{n} \sum_{j=1}^{m} j \cdot a_{i, j} & \leq m \cdot P \\
\sum_{i=1}^{n}\left(k_{i} \cdot P+\frac{u_{i}-\gamma_{i, k_{i}}}{\gamma_{i, k_{i}+1}-\gamma_{i, k_{i}}} \cdot P\right) & \leq m \cdot P \\
\sum_{i=1}^{n}\left(k_{i}+\frac{u_{i}-\gamma_{i, k_{i}}}{\gamma_{i, k_{i}+1}-\gamma_{i, k_{i}}}\right) & \leq m
\end{aligned}
$$

which is the claim of our theorem.

Theorem 5. Given any feasible task system $\tau$, the canonical schedule meet all the deadlines.

Proof. The proof consists of three parts: we first give an algorithm which constructs a schedule $\sigma$ for $\tau$, then we prove that $\sigma$ is canonical, and we finish by showing that the tasks meet their deadlines if $\tau$ is feasible.

The algorithm works as follows (see Algorithm 1): we consider sequentially every task $\tau_{i}$, with $i=n, n-1, \ldots, 1$ and define the schedule for these tasks in the time interval $[0,1)$, which is then repeated.

We calculate the duration (time interval) for which a task $\tau_{i}$ uses $k_{i}+1$ processors. If we denote by $\ell_{i}$ the duration that the task $\tau_{i}$ spends on $k_{i}+1$ processors, then we obtain the following equation:

$$
\ell_{i} \gamma_{i, k_{i}+1}+\left(1-\ell_{i}\right) \gamma_{i, k_{i}}=u_{i}
$$

Therefore we assign a task $\tau_{i}$ to $k_{i}+1$ processors for a duration of

$$
\frac{u_{i}-\gamma_{i, k_{i}}}{\gamma_{i, k_{i}+1}-\gamma_{i, k_{i}}}
$$

and to $k_{i}$ processors for the remainder of the interval, which ensures that the task satisfies its deadline, since each job generated by the sporadic task $\tau_{i}$ which arrives at time $t$ receives in the time interval $\left[t, t+T_{i}\right)$ exactly $T_{i} \times u_{i}=C_{i}$ time units.

The task $\tau_{n}$ is assigned to the processors $\left(p_{m}, \ldots, p_{m-k_{n}}\right)$ (see Figure 2). If $u_{n} \neq \gamma_{n, k_{n}+1}$, another task can be scheduled at the end of the interval on the processor $p_{m-k_{n}}$, as $\tau_{n}$ does not require $k_{n}+1$ processors on the whole interval.

We continue to assign greedily every task $\tau_{i}$, by first considering the processors with highest number. The schedule produced by the above algorithm is canonical as it respects the three constraints of the definition:

- on every processor $j$ we assign tasks by decreasing index, thus $\sigma_{j}(t)$ is monotone and decreasing;

- for all $i<i^{\prime}$, if $\tau_{i^{\prime}}$ is scheduled on a processor $p_{j^{\prime}}$, then $\tau_{i}$ is assigned to a processor $p_{j}$ with $j \leq j^{\prime}$;

- the schedule is repeated every unit of time. 


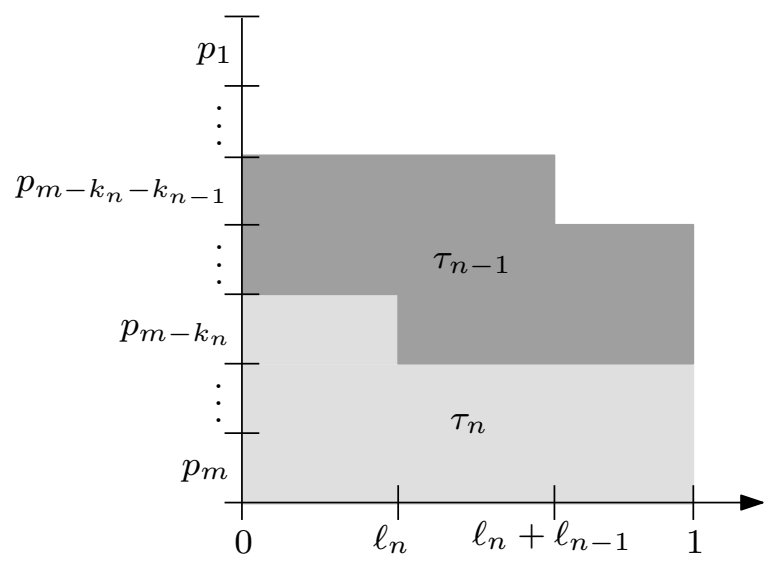

Figure 2: Schedule obtained after scheduling the task $\tau_{n}$

The last step is to prove that if our algorithm fails to construct a schedule, i.e., if at some point we run out of processors while there are still tasks to assign, then the system is infeasible.

Let $\lambda_{i}$ be the the total processor use of the task $\tau_{i}$ in every unit length time interval. In the case of a canonical schedule, $\lambda_{i}$ corresponds to:

$$
\lambda_{i}=k_{i}+\frac{u_{i}-\gamma_{i, k_{i}}}{\gamma_{i, k_{i}+1}-\gamma_{i, k_{i}}}
$$

So for instance if, in the canonical schedule, a task $\tau_{i}$ is assigned to $\lambda_{i}=2.75$ processors, it means that it is scheduled on two processors for 0.25 time unit in any time interval of length 1 , and on three processors for 0.75 time unit in the same interval.

If our algorithm fails, it means that $\sum_{i=1}^{n} \lambda_{i}>m$, which by Theorem 4 implies that the system is infeasible.

A detailed description of the scheduling algorithm is given by Algorithm 1 1 . If we consider the task system $\tau=\left\{\tau_{1}, \tau_{2}\right\}$ given before we have $k_{1}=1$ and $k_{2}=0$. By using Algorithm 1 we obtain:

$$
\begin{aligned}
& \sigma_{3}(t)=2, \forall t \in[0,0.75) \\
& \sigma_{3}(t)=1, \forall t \in[0.75,1) \\
& \sigma_{2}(t)=1, \forall t \in[0,1) \\
& \sigma_{1}(t)=1, \forall t \in[0,0.75) \\
& \sigma_{1}(t)=0, \forall t \in[0.75,1) .
\end{aligned}
$$

Notice that Algorithm 1 does not provide satisfactory schedules for problems for which the number of migrations and preemptions is an issue. We shall address this question in the next section.

Corollary 6. In the work-limited parallelism model and using an off-line scheduling algorithm, a necessary and sufficient condition for a sporadic task system $\tau$ to be feasible on $m$ processors is given 


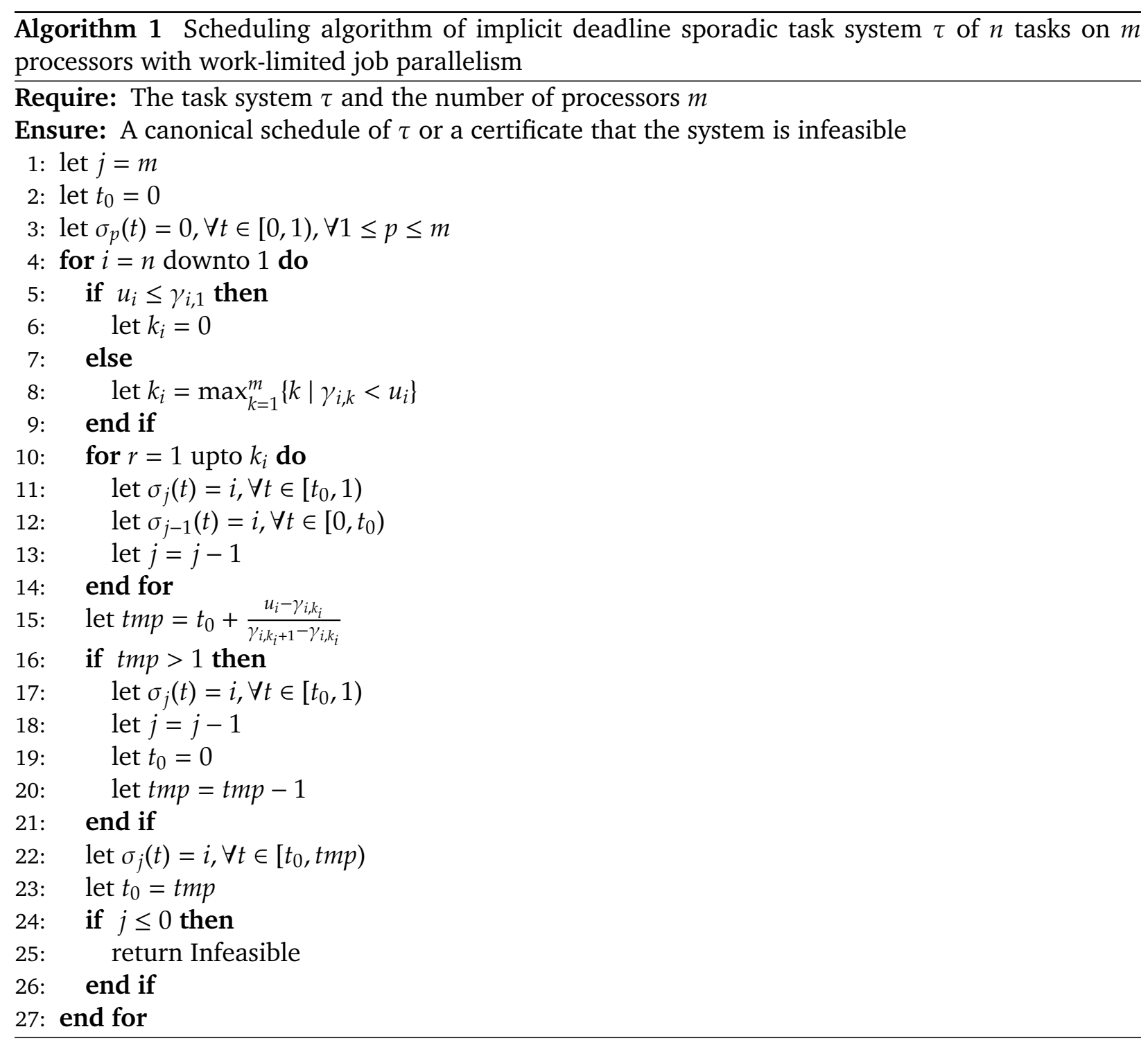


by:

$$
\sum_{i=1}^{n}\left(k_{i}+\frac{u_{i}-\gamma_{i, k_{i}}}{\gamma_{i, k_{i}+1}-\gamma_{i, k_{i}}}\right) \leq m
$$

Please notice that Corollary 6 can be seen as feasibility utilization bound and in particular a generalization of the bound for uniprocessor (see [12]) where a sporadic and implicit deadline task system is feasible if and only if $\sum_{i=1}^{n} u_{i} \leq 1$. Like the EDF optimality for sporadic implicit deadline tasks is based on the fact that $\sum_{i=1}^{n} u_{i} \leq 1$ is a sufficient condition, we prove the optimality of the canonical schedule based on the fact that $\sum_{i=1}^{n}\left(k_{i}+\frac{u_{i}-\gamma_{i, k_{i}}}{\gamma_{i, k_{i}+1}-\gamma_{i, k_{i}}}\right) \leq m$ is a sufficient condition.

Corollary 7. There exists an algorithm which, given any task system, constructs the canonical schedule or answers that no schedule exists in $O(n)$ time.

Proof. We know that the algorithm exists as it was used in the proof of Theorem 5. For every task, we have to compute the number of processors required (in $O(1)$ time, as the number of processors $m$ is fixed), and for every corresponding processor $j$, define $\sigma_{j}(t)$ appropriately. In total, $O(n)$ time is required.

\section{Scheduling problem reduction}

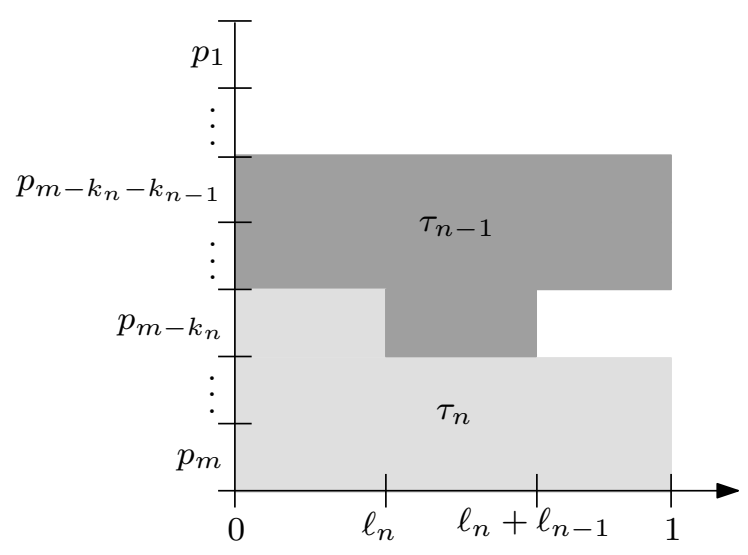

Figure 3: Improved scheduling of $\tau_{n-1}$ and $\tau_{n}$

Regarding optimality, we proved that each task (say $\tau_{i}$ ) must use permanently $k_{i}$ processor[s] simultaneously, and that optionally $\tau_{i}$ has to use an additional processor partially (i.e., with a duration strictly less than the unity in each interval of length 1 ). Regarding the number of migrations, we can however define a better schedule without loss of optimality. For instance, in the schedule given by Figure 2 , task $\tau_{n-1}$ migrates between $k_{n-1}+1$ processors each time unit. I.e., $\tau_{n-1}$ uses $k_{n-1}$ processors in $\left[0, \ell_{n}\right), k_{n-1}+1$ processors in $\left[\ell_{n}, \ell_{n}+\ell_{n-1}\right]$ and again $k_{n-1}$ processors in $\left[\ell_{n}+\ell_{n-1}, 1\right)$, but not the very same processors as the ones used in $\left[0, \ell_{n}\right]$. Consequently, there is necessarily a job migration of $\tau_{n-1}$ each time unit. We can however assign $k_{n-1}$ processors statically and permanently 
to $\tau_{n-1}$ and an additional processor sporadically (for a duration of $\ell_{n-1} \cdot T_{n-1}$, see Figure 3). In terms of optimality we use exactly the same number of processors but the number of migrations is null (at least for $\tau_{n-1}$ in this example). Since we assign statically and permanently tasks to processors we can actually reduce our scheduling problem: the scheduling problem of the $n$ (original) sporadic tasks upon $m$ processors with work-limited parallelism can be reduced to a more studied and simpler scheduling problem: the scheduling of $n^{\prime}\left(n^{\prime} \leq n\right)$ sporadic tasks upon $m^{\prime}\left(m^{\prime} \leq m\right)$ processors where job parallelism can be forbidden (without loss of optimality).

We shall now formalize the scheduling problem reduction. Let $\left\{\tau_{1}, \tau_{2}, \ldots, \tau_{n}\right\}$ be our (original) sporadic task set to schedule on $m$ processors. By the definition of the quantity $k_{i}$ (Eq. 1) and the optimality of Algorithm 2 (Theorem 5), the scheduling problem can be reduced to the scheduling of the sporadic task set $\tau^{\prime} \stackrel{\text { def }}{=}\left\{\tau_{1}^{\prime}, \tau_{2}^{\prime}, \ldots, \tau_{n}^{\prime}\right\}$ with $C_{i}^{\prime} \stackrel{\text { def }}{=} \ell_{i} \cdot T_{i}, T_{i}^{\prime} \stackrel{\text { def }}{=} T_{i}$ upon $m^{\prime} \stackrel{\text { def }}{=} m-\sum_{i=1}^{n} k_{i}$ where job parallelism can be forbidden (without loss of optimality).

While our (canonical) schedules are optimal (the original one and the improved one) for $\tau$, the number of preemptions can be too large (there is actually, at least one preemption each time unit for the set of tasks $\tau^{\prime}$ ). For the systems where this is an issue, since job parallelism can be forbidden for $\tau^{\prime}$, with potential loss of schedulability, we can schedule $\tau^{\prime}$ using, for instance, global EDF (see [7]), global Rate Monotonic (see [2]) or using a partition scheme (see [1] for instance) in order to reduce nicely the number of preemptions. We shall analyze more precisely the case of using EDF to schedule the task sub-set $\tau^{\prime}$ in the next section.

\subsection{Scheduling $\tau^{\prime}$ using EDF}

In order to reduce strongly the number of preemptions in the schedule of task sub-set $\tau^{\prime}$ we can use EDF (or one of its variants) instead of the canonical schedule. We know that the number of preemptions using EDF is bounded from above by the number of jobs in the set (and consequently, the total number of context switches is bounded by twice the number of jobs). It can similarly be shown that the total number of interprocessor migrations of individual jobs is bounded from above by the number of jobs. We know that the task sub-set $\tau^{\prime}$ is schedulable using the optimal canonical schedule since the following necessary and sufficient condition is satisfied:

$$
\sum_{\tau_{i}^{\prime} \in \tau^{\prime}} u_{i}^{\prime} \leq m^{\prime}
$$

Unfortunately, EDF is not optimal in terms of the number of processors required and the condition above is only necessary. Using, e.g., EDF-US[1/2] (which gives top priority to jobs of tasks with utilizations above 1/2 and schedules the remaining jobs using EDF) Baker [3] proved correct the following sufficient (not exact) feasibility test: the task sub-set $\tau^{\prime}$ is schedulable by EDF-US [1/2] upon $\widehat{m}$ processors if

$$
\left(2 \cdot \sum_{\tau_{i}^{\prime} \in \tau^{\prime}} u_{i}^{\prime}-1\right) \leq \widehat{m}
$$

The trade-off is actually the additional number of processors required: $\left[2 \cdot \sum_{\tau_{i}^{\prime} \in \tau^{\prime}} u_{i}^{\prime}-1\right\rceil-m^{\prime}$. 


\section{Discussions}

\subsection{Job parallelism vs. task parallelism}

In this manuscript we study multiprocessor systems where job parallelism is allowed. We would like to distinguish between two kinds of parallelism, but first the definitions: task parallelism allows each task to be executed on several processors at the same time, while job parallelism allows each job to be executed on several processors at the same time. If we consider constrained (or implicit) deadline systems task parallelism is not possible. For arbitrary deadline systems, where several jobs of the same task can be active at the same time, the distinction makes sense. Task parallelism allows the various active jobs of the same task to be executed on a different (but unique) processor while job parallelism allows each jobs to be executed on several processors at the same time.

\subsection{Optimality and future work}

In this paper we study the feasibility problem of implicit deadline sporadic task systems with worklimited job parallelism upon identical parallel machines when global scheduling is used. We prove that the time-complexity of our problem is linear relative to the number of tasks. We provide an optimal scheduling algorithm that runs in $O(n)$ time and we give an exact feasibility utilization bound.

Our algorithm is optimal in terms of the number of processors used. It is left open whether an optimal algorithm in terms of the number of preemptions can be designed. As a first step, we used an interval of length 1 to study the feasibility problem. Without loss of optimality, we can improve our algorithm to work on an interval of length equal to the $\mathrm{gcd}$ of the periods of every task, which decreases the number of preemptions and migrations. We do not know, however, if this improvement minimizes the number of preemptions and migrations.

The definition of work-limited job parallelism was given here for identical processors. One should investigate an extension of this definition to heterogeneous platforms.

\section{Acknowledgments}

The authors thank anonymous referees, whose remarks led us to better present our results. We also thank Ben Rodriguez for interesting discussions on potential extensions of this work, as well as for key ideas to fix the proof of Theorem 4 .

\section{References}

[1] B. Andersson, Static - priority scheduling on multiprocessors, Ph.D. thesis, Chalmers University of Technology, Göteborg, Sweden (2003).

[2] B. Andersson, S. K. Baruah, J. Jonsson, Static-priority scheduling on multiprocessors, Proceedings of the 22nd IEEE Real-time Systems Symposium (2001) 193-202.

[3] T. P. Baker, An analysis of EDF scheduling on a multiprocessor, IEEE Trans. on Parallel and Distributed Systems 15 (8) (2005) 760-768. 
[4] T. P. Baker, S. K. Baruah, Handbook of Real-Time and Embedded Systems, chap. Schedulability Analysis of Multiprocessor Sporadic Task Systems, Chapman and Hall, 2007, pp. 3-1 3-15.

[5] R. Chandra, L. Dagum, D. Kohr, D. Maydan, J. McDonald, R. Menon, Parallel programming in OpenMP, Morgan Kaufmann Publishers Inc., San Francisco, CA, USA, 2001.

[6] A. Geist, A. Beguelin, J. Dongarra, W. Jiang, R. Manchek, V. Sunderam, PVM: Parallel Virtual Machine A Users' Guide and Tutorial for Networked Parallel Computing, MIT Press, 1994.

[7] J. Goossens, S. Funk, S. K. Baruah, Priority-driven scheduling of periodic task systems on multiprocessors, Real-time Systems 25 (2-3) (2003) 187-205.

[8] S. Gorlatch, H. Bischof, A generic MPI implementation for a data-parallel skeleton: Formal derivation and application to FFT, Parallel Processing Letters 8 (4) (1998) 447-458.

[9] W. Gropp (ed.), Using MPI: portable parallel programming with the message-passing interface, 2nd ed., Cambridge, MIT Press, 1999.

[10] C. Han, K.-J. Lin, Scheduling parallelizable jobs on multiprocessors, Proceedings of the 10th IEEE Real-Time Systems Symposium (RTSS'89) (1989) 59-67.

[11] E. L. Leiss, Parallel and Vector Computing, McGraw-Hill, Inc., 1995.

[12] C. Liu, J. Layland, Scheduling algorithms for multiprogramming in a hard-real-time environment, Journal of the ACM 20 (1) (1973) 46-61.

[13] G. Manimaran, C. Siva Ram Murthy, K. Ramamritham, A new approach for scheduling of parallelizable tasks in real-time multiprocessor systems, Real-Time Systems 15 (1998) 39-60.

[14] A. Mok, Fundamental design problems of distributed systems for the hard-real-time environment, Ph.D. thesis, Laboratory for Computer Science, Massachusetts Institute of Technology (1983).

[15] A. Srinivasan, S. Baruah, Deadline-based scheduling of periodic task systems on multiprocessors, Information Processing Letters 84 (2002) 93-98.

[16] V. Sunderam, PVM: A framework for parallel distributed computing, Concurrency: Practice and Experience 2 (4) (1990) 315-339.

[17] A. Y. Zomaya, Parallel processing for real-time simulation: A case study, IEEE Parallel and Distributed Technology: System and Technology 4 (2) (1996) 49-55. 